\title{
HÁBITOS ALIMENTARIOS Y ESTADO EMOCIONAL DE LA POBLACIÓN DE DOS DISTRITOS DE LA REGIÓN TACNA, EN EL PERIODO DE AISLAMIENTO POR EL COVID - 19
}

\author{
EATING HABITS AND EMOTIONAL STATE OF THE POPULATION OF TWO \\ DISTRICTS OF THE TACNA REGION, IN THE PERIOD OF ISOLATION BY THE \\ COVID - 19
}

Gaby Yamilex Quispe Guerra ${ }^{1}$ Lucia Marianne Rodríguez Yunca²

Raul Cartagena Cutipa ${ }^{3}$

Información del artículo:

Recibido: 27/10/2020

Aceptado: 22/11/2020

${ }^{1}$ Escuela de Ingeniería Agroindustrial, Universidad Privada de Tacna, Perú ${ }^{3}$ Docente escuela de Ingeniería Agroindustrial, Universidad Privada de Tacna, Perú E-mail: uptgaby@gmail.com; Marianne150196@hotmail.com, raucartagenac@upt.pe 


\section{Resumen}

El objetivo del estudio fue determinar los efectos del aislamiento por el Covid-19 y su relación con los hábitos alimentarios en dos distritos de la región Tacna en el año 2020, identificando los efectos en la alimentación, consumo de alimentos, la búsqueda involuntaria de alimentos, comportamiento alimentario y la salud emocional de la población. La metodología es de tipo no experimental, transversal, la población estudiada estuvo compuesta por pobladores del distrito de Tacna (zona urbana) y del distrito de Locumba (zona rural), determinando muestras probabilísticas de 386 y 300 respectivamente, entre las edades de 18 a 64 años. Se empleó la técnica de encuesta, desarrollando un instrumento con una fiabilidad 79,4 \% (alfa de Cronbach) que constan de 19 preguntas que fueron categorizadas en dimensiones y analizadas mediante pruebas no paramétricas de Chi cuadrado. Los resultados indican que los cambios en los hábitos alimentarios están relacionados con el aislamiento por el Covid-19 en los dos distritos con un nivel de confianza de $95 \%$, además al comparar los cambios debido a la pandemia en los dos distritos, se encontró que el distrito de Locumba tuvo los mayores efectos. Por lo tanto, se concluye que si existió efectos causados por el aislamiento por el Covid-19 sobre los hábitos alimentarios en los dos distritos de la región Tacna.

Palabras clave: Hábitos alimentarios; aislamiento por Covid 19; salud emocional.

\section{Abstract}

The objective of the study was to determine the effects of Covid-19 isolation and its relationship to eating habits in two districts of the Tacna region in 2020, identifying the effects on food, food consumption, involuntary food search, food behavior and emotional health of the population. The methodology is non-experimental, transverse, the population studied was composed of settlers from the district of Tacna (urban area) and the district of Locumba (rural area), determining probability samples of 386 and 300 respectively, between the ages of 18 to 64 years. The survey technique was used, developing an instrument with 79.4\% reliability (Cronbach alpha) consisting of 19 questions that were categorized into dimensions and analyzed using non-parametric tests of Chi squared. The results indicate that changes in eating habits are related to Covid-19 isolation in the two districts with a $95 \%$ confidence level, and when comparing changes due to the pandemic in the two districts, Locumba district was found to have the greatest effects. It is therefore concluded that if there were effects caused by Covid-19 isolation on eating habits in the two districts of the Tacna region.

Key words: Eating habits; insulation by Covid 19; emotional health. 


\section{Introducción}

En marzo de 2020, la Organización Mundial de la Salud (OMS) declaró la enfermedad por Coronavirus como una pandemia (OMS, 2020). La gran mayoría de países pusieron en acción medidas restrictivas como prohibiciones de viajes y cierres para prevenir la propagación de la infección. Desafortunadamente, muchas personas entraron en pánico por comprar y abastecerse de alimentos, ya que el momento adecuado para el control de la pandemia es impredecible.

El efecto que trajo consigo las crisis de COVID-19 en cuanto al comportamiento referidos a los hábitos alimentarios que incluye una alimentación sana y el ejercicio físico, no es tan claro, pero puede llegar a ser algo sustancial (Pearl, 2020). En el transcurso que se fue desarrollando la pandemia, hubo suspensión de la cadena de suministros, lo cual llevó a que las personas realicen las compras de pánico lo que lleva a un poco acceso a los productos frescos, esto lleva al aumento del consumo de alimentos de vida útil larga (Tan et al., 2020). Las personas al ser forzadas a permanecer dentro de sus casas debido a la pandemia, durante un periodo largo pueden llegar a desencadenar estrés lo cual causa un cambio total en sus hábitos alimentarios.

El estudio busca conocer los efectos causados por el aislamiento por COVID-19 sobre la alimentación, si en la alimentación se vio cambios o no durante la pandemia, teniendo como objetivo investigar los "efectos del aislamiento y su relación con los hábitos alimentarios en dos distritos de la región Tacna", teniendo en consideración que Tacna es la región con más altos índices de sobrepeso y obesidad en el país (Gobierno regional de Tacna, 2020). Para el desarrollo del estudio se analizó el aumento del consumo de alimentos, la búsqueda involuntaria de alimentos y el comportamiento frente al consumo de alimentos, factores que influyen en la alimentación de la población durante el aislamiento basándose en alimentos poco saludables, poco nutritivos y que son de fácil acceso y de consumir.

\section{Objetivos}

Determinar la relación entre los hábitos alimentarios y el estado emocional de la población de dos distritos de la región Tacna en el periodo de aislamiento por Covid 19.

\section{Metodología}

Se realizó un estudio no experimental y transversal, en una muestra representativa de dos distritos Locumba y Tacna de personas entre las edades de 18 a 64 años de edad, la técnica empleada para la recogida de la información, y dada la situación que se generó por el brote del COVID-19, se realizó mediante un cuestionario en Google Forms, que se mediante medios virtuales, esta modalidad fue complementada con entrevistas físicas. En el estudio participaron 686, siendo 300 participantes del distrito de Locumba y 386 del distrito de Tacna una vez saneada la base de datos, se realizó el análisis de las respuestas. 
Para medir los efectos sobre la bienestar emocional se aplicó la escala DASS que se estructuró tomando como referencia el cuestionario propuesto por Antunez y Vinet (2012), que está compuesta por 21 ítems con escalas tipo Likert. Las opciones de respuesta disponibles para responder a esta escala fueron: 0: no me ha ocurrido (nunca); 1: me ha ocurrido un poco (a veces); 2: me ha ocurrido bastante(a menudo), y 3: me ha ocurrido mucho (casi siempre). Para analizar los hábitos alimentarios se realizó una adaptación de la encuesta de Comportamiento Alimentarios de Alves et al., (2006), en el consumos de alimentos, búsqueda involuntaria de alimentos y el comportamiento alimentario frente al consumo de alimentos la cual ayuda a evaluar la frecuencia, cantidad, aumento de alimentos procesado y ultra procesados, alimentación antes y después del aislamiento utilizando la escala tipo Likert, se aplicó la siguiente escala; 4: Aumentó mucho,3: Aumento Poco, 2: No aumentó ni disminuyó, 1: Disminuyó poco, 0: Disminuyó mucho. Para la determinación de la fiabilidad del instrumento se recurrió al alfa de Cronbach que resultó con un índice de fiabilidad de $79,4 \%$, siendo muy aceptable para este tipo de estudios y específico para testear las escalas de medida planteadas.

\section{Resultados}

En la tabla 1 se muestra una tabla de doble entrada que relaciona los hábitos alimentarios y la salud emocional en los dos distritos, al hacer un contraste estadístico donde se muestra que la población cambio sus hábitos alimentarios y la salud emocional y ambas variables se relacionan significativamente $(X 2=78,794 ; \mathrm{p}<0 ; 05)$ con un nivel de confianza del $95 \%$, en Tacna se observó que la población cambió "Bastante" sus hábitos alimentarios representados por un 23,1\%, mientras que en su estado emocional se observó cambios "A veces" con un 35,3\%. Mientras que en Locumba se puede observar que el $37,0 \%$ de los encuestados cambiaron "Bastante" en sus hábitos alimentarios, el $19,3 \%$ tuvo cambios en su estado emocional. Realizando una comparación en los dos distritos se puede concluir que en Locumba hubo más cambios en los hábitos alimentarios, por otro lado, en el distrito de Tacna es donde se presentan mayores cambios en el estado emocional de las personas durante el aislamiento por el Covid-19.

En la Tabla 2. Se muestra el aumento del consumo de alimentos durante la pandemia, donde las dos poblaciones tuvieron un aumento significativo, se evidencia que 50,6 \% en el distrito de Tacna y el $81,6 \%$ del distrito de Locumba aumentaron el consumo de alimentos, realizando una comparación entre los dos distritos se puede concluir que en la población de Locumba hubo más consumo de alimentos $(x 2=47,261 ; p<0,01)$, siento estadísticamente significativo al $95 \%$. 
Tabla 1

Estado emocional en relación a los cambio de hábitos alimentarios de la población de Tacna y locumba

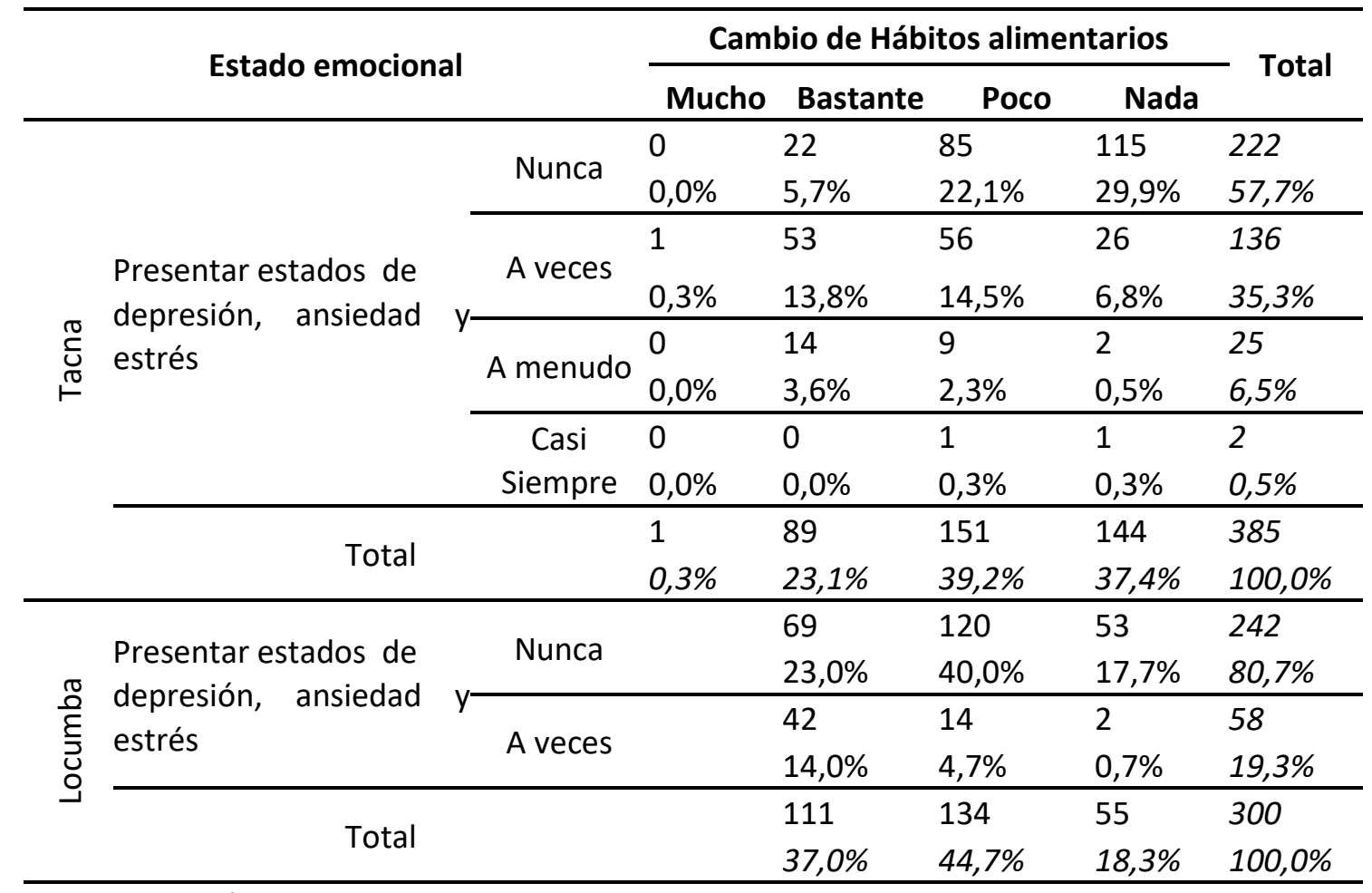

Nota. Elaboración propia.

Tabla 2

Aumento del consumo de alimentos y distintos de origen

\begin{tabular}{lccc}
\hline \multirow{2}{*}{ Nivel de aceptación } & \multicolumn{2}{c}{ Distrito de origen } & \multirow{2}{*}{ Total } \\
\cline { 2 - 3 } Definitivamente sí & Tacna & Locumba & \\
\hline \multirow{2}{*}{ Probablemente sí } & 102 & 124 & 226 \\
& $26,4 \%$ & $41,3 \%$ & $32,9 \%$ \\
\hline \multirow{2}{*}{ Probablemente no } & 119 & 121 & 240 \\
& $30,8 \%$ & $40,3 \%$ & $35,0 \%$ \\
\hline \multirow{2}{*}{ Definitivamente no } & 118 & 41 & 159 \\
& $30,6 \%$ & $13,7 \%$ & $23,2 \%$ \\
\hline \multirow{2}{*}{ Total } & 47 & 14 & 61 \\
& $12,2 \%$ & $4,7 \%$ & $8,9 \%$ \\
\hline
\end{tabular}

Nota. Elaboración propia.

La tabla 3 muestra las frecuencias de la búsqueda involuntaria de alimento por el aislamiento, evidenciando que las poblaciones de los dos distritos sintieron deseos de comer ello podría ocasionar la búsqueda involuntaria de alimentos que los satisfagan. En cuanto al distrito de Locumba representado con el $81,7 \%$ y 50,6 \% de la población del distrito de Tacna, "definitivamente si" o "probablemente si" piensan o sienten deseos de comer, comparando los dos distritos se puede concluir que en el distrito de Locumba sintieron mayor deseo de comer, y que estadísticamente las dos variables se relacionan $(x 2=72,988 ; p<0,01)$ con un nivel de confianza del $95 \%$. 
Tabla 3

Tabla de contingencia piensa o siente deseos de comer

\begin{tabular}{lccc}
\hline \multirow{2}{*}{ Nivel de aceptación } & \multicolumn{2}{c}{ Distrito de origen } & \multirow{2}{*}{ Total } \\
\cline { 2 - 3 } & Tacna & Locumba & \\
\hline \multirow{2}{*}{ Definitivamente sí } & 72 & 95 & 167 \\
& $18,7 \%$ & $31,7 \%$ & $24,3 \%$ \\
\hline \multirow{2}{*}{ Probablemente sí } & 123 & 150 & 273 \\
& $31,9 \%$ & $50,0 \%$ & $39,8 \%$ \\
\hline \multirow{2}{*}{ Probablemente no } & 129 & 43 & 172 \\
\cline { 1 - 2 } Definitivamente no & $33,4 \%$ & $14,3 \%$ & $25,1 \%$ \\
\hline \multirow{2}{*}{ Total } & 62 & 12 & 74 \\
& $16,1 \%$ & $4,0 \%$ & $10,8 \%$ \\
\hline
\end{tabular}

Nota. Elaboración propia

En la tabla 4 se muestra el comportamiento frente al consumo de alimentos por el aislamiento, en donde indica que la población de los distritos en general si tuvo cambios en la frecuencia que come fuera de control. Asimismo, se muestra que en la población de Locumba el $54,7 \%$ y Tacna con $42 \%$ afirman que con frecuencia comen fuera de control, concluyendo que la población de Locumba tiene más tendencia a comer fuera de control, por otro lado existiría una relación significativa de ambas variables $(x 2=78,062 ; p<0,01)$ al $95 \%$ de confianza.

Tabla 4

Frecuencia que come fuera de control durante el aislamiento

\begin{tabular}{lccc}
\hline \multirow{2}{*}{ Frecuencia de realización } & \multicolumn{2}{c}{ Distrito de origen } & \multirow{2}{*}{ Total } \\
\cline { 2 - 3 } & Tacna & Locumba & \\
\cline { 1 - 3 } Siempre & 21 & 2 & 23 \\
& $5,4 \%$ & $0,7 \%$ & $3,4 \%$ \\
\hline \multirow{2}{*}{ Casi siempre } & 72 & 99 & 171 \\
& $18,7 \%$ & $33,0 \%$ & $24,9 \%$ \\
\hline \multirow{2}{*}{ A veces } & 138 & 158 & 296 \\
& $35,8 \%$ & $52,7 \%$ & $43,1 \%$ \\
\hline \multirow{2}{*}{ Casi Nunca } & 155 & 41 & 196 \\
& $40,2 \%$ & $13,7 \%$ & $28,6 \%$ \\
\hline \multirow{2}{*}{ Total } & 386 & 300 & 686 \\
& $100,0 \%$ & $100,0 \%$ & $100,0 \%$ \\
\hline
\end{tabular}

Nota. Elaboración propia

\section{Discusiones}

\section{Efectos sobre el bienestar emocional}

El estado emocional de los participantes es un factor muy importante y determinante al momento de consumir los alimentos, en esa dirección se realizó una evaluación sobre la bienestar 
emocional obteniéndose que el $67,7 \%$ de los participantes no presentó en gran medida cambios en la salud emocional, sin embargo, el 32,3 \% que representa un tercio de los participantes si presentaron cambios en la salud emocional presentando niveles moderado a altos de estrés, ansiedad y depresión.

La relación entre las emociones y la conducta alimentaria depende de la emoción que presente la persona de acuerdo a Palomino (2020), quien refiere que el individuo emplea alimentos como una forma de afrontar las emociones negativas, puede conllevar al aumento del consumo de los "alimentos reconfortantes". En los resultados del estudio se destaca que las poblaciones de los dos distritos presentaron efectos significativos en su salud emocional, esto va de acuerdo con Barattucci (2011) quien encontró que las personas que más comen son las que atraviesan situaciones de estrés, más ansiosos o deprimidos y que tienden a recurrir al consumo de alimentos como medio de escape. Respecto a la afectación se encontró que en los niveles "nunca" destaca el $67,7 \%$, mientras que en el grupo de "a veces hasta casi siempre" suman en total 32,3\%, esto haría notar que de alguna forma presentan situaciones negativas en su salud emocional. Para contrastar estadísticamente, se efectuó la prueba de Chi cuadrado, donde se comprueba que efectivamente sí hubo cambios en la salud emocional de la población, al comparar por distritos se encontró que la población de Tacna tuvo mayores efectos en la salud emocional ( $p$-valor $<0,01$ ) al $95 \%$ de confianza. Este resultado se explicaría a las restricciones que fueron impuestas por el gobierno, tales como el aislamiento social que conllevo a distintas restricciones.

\section{Hábitos alimentarios}

El consumo de alimentos aumentó significativamente durante el aislamiento, observando que el $67,9 \%$ de los participantes aumentaron su consumo de alimentos y en su mayoría alimentos poco saludables, destacándose una tendencia clara al aumento de consumo de productos orneados y snack y otro grupo de población opto más bien por alimentos frescos o procesados, estas variaciones se explicarían por el hecho que las personas eligen los alimentos según el estado emocional, esta posición es compartida con Cheikh Ismail et al., (2020) quienes indican que las nuevas tendencias en los comportamientos alimentarios son negativos durante el aislamiento, como es la ingesta de productos con mayores calorías. En ese sentido en el estudio se encontró que la población de los dos distritos presentó efectos significativos en el cambio del consumo de alimentos, observándose un aumento de consumo en los productos horneados. Así mismo el estudio de Robinson et al. (2020) sobre obesidad y conducta alimentaria durante la pandemia, reportó que una salud mental negativa se asocia con un aumento de consumo en exceso, tal cual ocurre en la situación actual en donde el 67,9 \% aumentó el consumo de alimentos en la población de los distritos estudiados, también se encontró cambios en la forma de consumir sus alimentos, ya que el $17,2 \%$ indica que come por aburrimiento, como lo afirma Santangelo y Borg (2020), el mayor cambio en el comportamiento alimentario es comer por aburrimiento. Al realizar un contraste estadístico se comprueba que efectivamente sí hubo un aumento en el consumo de alimentos, mientras que al comparar por distritos se encontró que la población de Locumba tuvo mayores efectos ( $p$-valor $=0.01$ )en el consumo de alimentos al $95 \%$ de confianza, este resultado se debería a que los pobladores de la zona rural por su actividad laboral (agrícola) requieren más cantidad del consumo de alimentos ya que realizan más esfuerzo físico durante el día, así con la actual pandemia 
este consumo aumentó involuntariamente por motivos de estrés y preocupación por parte de la población, tal como evidencian los resultados.

La búsqueda involuntaria durante el aislamiento también aumentó significativamente, encontrándose que el 64,1 \% de la población manifestó que siente grandes deseos de comer, en consecuencia ingieren más alimentos en comparación a sus hábitos normales antes de la llegada de la pandemia, en consecuencia las personas al pasar por emociones negativas llegan a consumir alimentos involuntariamente, como lo indica Vergara (2020) quien afirma que buscar involuntariamente los alimentos se debe a que la persona pasa por una modificación inesperada en su estilo de vida, en los resultado de la investigación, la población de los dos distritos presentaron cambios en su alimentación, buscando un refugio en la comida, como lo indica Smink (2020) que las personas no toleran el malestar psicológico y tienden a refugiarse en la intercomunicación social, lo cual no lo realizaron debido a la cuarentena, por refugiarse en los alimentos. Según Vivas (2020) sobre los patrones de consumo en la alimentación ante la pandemia COVID-19 encontró cambios significativos en la alimentación de los bogotanos, tal cual ocurre en la situación que se vive en la región donde se observó un cambio y aumento en la alimentación, cantidad de comidas, frecuencia de consumo, consumo de alimentos fuera de control durante el aislamiento, en concordancia con el estudio de Bebenek et al., (2020) reportaron que el número de comidas consumidas durante el aislamiento aumentó significativamente, de igual forma ocurre con Ammer et al., (2020) que reporta que la alimentación durante el aislamiento es poco saludable. Al realizarse un contraste estadístico se corrobora que sí hubo cambios en la alimentación de la población. Mientras que al comparar por distritos se encontró que la población de Locumba tuvo mayores efectos ( $p$-valor $=0,01$ ) en la alimentación al 95 \% de confianza, se debería a que lo pobladores de la zona rural gastan más energía a lo largo del día, ya que se dedican a diversos trabajos agrícolas y ganaderas, el consumo involuntario se debe a que existe preocupación sobre el futuro de sus trabajos, la venta de sus productos que influirían al consumo de más alimentos y poco saludables.

Otro aspecto evaluado fue la frecuencia de consumo alimentos fuera de control, que podría generar desequilibrio en la salud, este aspecto podría acentuarse por el confinamiento que atraviesa la población, que estaría provocando consumo desmedido de alimentos poco saludables, al respecto el 28,3\% ingiere con mayor frecuencia sus alimentos, mientras que se muestra que el $43,1 \%$ a veces ingiere algunos alimentos en exceso. Las emociones negativas son factores que influyen en la alimentación y se relacionan con los hábitos alimentarios, según Pérez et al., (2020) refiere que el individuo puede generar emociones negativas tales como es la ansiedad, enojo, las cuales contribuyen a alterar la alimentación en cuanto a la cantidad, variedad y frecuencia de consumo. También Severi y Medina (2020) revelan que la tercera parte de la población uruguaya indicó haber realizado cambios en su alimentación y que $47 \%$ come con mayor frecuencia, los resultados de la investigación se destaca que el $49,9 \%$ de la población de los dos distritos come fuera del control con mayor frecuencia, además que tienen más hambre entre las comidas principales estos resultados van en concordancia con Gutiérrez (2020) que menciona que los pensamientos y emociones negativas generan comportamiento alimentarios negativos, como comer con mayor frecuencia $y$ alimentos bajos en nutrientes. Al realizarse un contraste estadístico, se comprueba que sí hubo cambios en el comportamiento frente al consumo de alimentos. Mientras que al comparar por distritos se encontró que la población de Locumba tuvo mayor frecuencia del consumo fuera de control al 95\% de confianza. 


\section{Conclusiones}

Existe una relación significativa entre los efectos del aislamiento por el COVID-19 y los hábitos alimentarios de la población en los dos distritos de la región Tacna en el año 2020, siendo que el distrito de Locumba el $81,7 \%$ y Tacna el $62,6 \%$ cambiaron sus hábitos alimentarios a causa del aislamiento.

El estado emocional es importante a la hora de afrontar el COVID-19, por lo que se concluye que los niveles de afectación respecto a la salud emocional fueron significativos, además en los patrones, aumentó el consumo de los alimentos respecto a sus hábitos alimentarios habituales, destacándose los cambios en la forma de la elección de alimentos, cantidades de consumo al día. Se evidencia cambios significativos en la búsqueda involuntaria de alimentos de la población de los distritos de Tacna y Locumba donde la población presenta más deseos de comer, aumentando el consumo de los alimentos con respecto a la alimentación antes del aislamiento, demostrándose además que la población de Locumba con un 81,7 \% tubo más inclinación a buscar y consumir más alimentos, en comparación con el distrito de Tacna.

Finalmente, el comportamiento frente al consumo de alimentos en estado de aislamiento, mostró que la población incurre con más frecuencia a comer fuera de control, destacándose que el $54,7 \%$ de la población de Locumba presenta los mayores efectos.

\section{Referencias Bibliográficas}

Alves, J., Cruz, A., \& Aguiar, Z. (2006). Adaptación para la lengua portuguesa de la depression, anxiety and stress scale (DASS). Scielo.br. Retrieved 14 November 2020, from https://www.scielo.br/pdf/rlae/v14n6/es_v14n6a06.pdf.

Antúnez, Z., y Vinet, E. (2012). Escalas de Depresión, Ansiedad y Estrés (DASS - 21): Validación de la Versión abreviada en Estudiantes Universitarios Chilenos. Terapia psicológica, 30(3), $49-55$. https://dx.doi.org/10.4067/S0718-48082012000300005

Gobierno Regional de Tacna (2020). Estrategia De Prevención Sanitaria Y Socioeconómica Para Hacer Frente

Al

Covid-19. http://ww2.regiontacna.gob.pe/grt/documentos/2011/personal/ESTRATEGIA SCORAPREC.pdf

OMS, (2020). Nuevo Coronavirus 2019. [online] Who.int. Available at: <https://www.who.int/es/emergencies/diseases/novel-coronavirus-2019>

Palomino, A., 2020. Rol De La Emoción En La Conducta Alimentaria. [online] Available at: <http://dx.doi.org/10.4067/S0717-75182020000200286>

Pearl, R. (2020). El estigma del peso y la "cuarentena-15" https://doi.org/10.1002/oby.22850

Barattucci, Y., 2011. Estrés Y Alimentación. Core.ac.uk. Available at: https://core.ac.uk/download/pdf/49224352.pdf

Cheikh Ismail, L., Osaili, T., Mohamad, M., Al Marzouqi, A., Jarrar, A., Abu Jamous, D., Magriplis, E., Ali, H., Al Sabbah, H., Hasan, H., AlMarzooqi, L., Stojanovska, L., Hashim, M., Shaker Obaid, R., Saleh, S. and AI Dhaheri, A., 2020. Eating Habits and Lifestyle during COVID-19 Lockdown in the 
United Arab Emirates: A Cross-Sectional Study. Nutrients, 12(11), p.3314. https://www.mdpi.com/2072-6643/12/11/3314/htm\#B9-nutrients-1203314

Robinson, E., Boyland, E., Chisholm, A., Harrold, J., Maloney, N., Marty, L., Mead, B., Noonan, R., Hardman, C. (2020). Obesity, eating behavior and physical activity during COVID-19 lockdown: A study of UK adults, Appetite, Volume 156, 2021, 104853, https://doi.org/10.1016/j.appet.2020.104853

Santangelo, G. y Borg, A., 2020. Informe Comparativo: Hábitos Alimentarios Y De Actividad Física Durante El Aislamiento Social, Preventivo Y Obligatorio. Repositorio.uade.edu.ar. Available at: <https://repositorio.uade.edu.ar/xmlui/handle/123456789/11688>

Vergara, A., (2020). Qué Comer Antes, Durante Y Después De La Pandemia Por COVID-19. infobae. Available at: https://www.infobae.com/america/mexico/2020/06/02/que-comerantesdurante-y-despues-de-la-pandemia-por-covid-19/

Smink, V. (2020). Coronavirus en Argentina: los efectos que está teniendo la cuarentena más larga del mundo sobre los argentinos [Blog]. https://www.bbc.com/mundo/noticias-america-latina53857858.

Vivas, R., (2020). Patrones de consumo en la alimentación de los individuos de altos ingresos de Bogota, ante la pandemia COVID-19. Colegio de Estudios Superiores de Administración - CESA-. Bogota

Bebenek, E., Jgielsk, P., Boleslawska, I., Jagielsk, A., Osuch, A. y Kawalec P. (2020). Nutrition Behaviors in Polish Adults before and during COVID-19 Lockdown. https://doi.org/10.3390/nu12103084

Ammer, A., Brach, M., Trabelsi, K., Chtourou, H., Boukhris, O., Masmoudi, L., Bouaziz, B., Bentlage, E., How, D., Ahmed, M., Muller P., Muller, N., Aloui, A., Hammouda, O., Domingos, L., Braakman, A., Wrede, C., Bastoni, S., Pernambuco, C., Mataruna, L. y Hoekelmann, A.,1 on behalf of the ECLBCOVID19 Consortium (2020). Effects of COVID-19 Home Confinement on Eating Behaviour and Physical Activity: Results of the ECLB-COVID19 International Online Survey.

Pérez, C., Citores, M. G., Bárbara, G. H., Litago, F. R., Casis, L., Aranceta-bartrina, J., C., Arija, V., Ana, M., Victoria, E. M. De, Ortega, R. M., Partearroyo, T., \& Quiles, J. (2020). Cambios en los hábitos alimentarios durante el periodo de confinamiento por la pandemia COVID-19 en España Introducción La pandemia sanitaria mundial motivada por. 26(2). https://doi.org/10.14642/RENC.2020.26.2.5213

Tan, M., Feng, J. y Graham M. (2020). Obesidad y COVID-19: el papel de la industria alimentaria. https://doi.org/10.1136/bmj.m2237

Severi, C., \& Medina, M. (2020). La etología aplicada en el bienestar y productividad del cerdo en granja. porciNews, la revista global del porcino. Retrieved 18 November 2020, from https://porcino.info/la-etologia-aplicada-en-elbienestar-y-productividad-del-cerdo-en-granja/.

Gutiérrez, M. (2020). La salud mental y el aislamiento social en tiempos de COVID19. Revista.ibd.senado.gob.mx. Consultado el 18 de noviembre de 2020, en http://revista.ibd.senado.gob.mx/index.php/PluralidadyConsenso/article/view File/6. 